\title{
Article \\ In the Search of Marine Pestiviruses: First Case of Phocoena Pestivirus in a Belt Sea Harbour Porpoise
}

\author{
Iben Stokholm ${ }^{1,2}{ }^{\oplus}$, Nicole Fischer ${ }^{3}(0)$, Christine Baechlein ${ }^{4}$, Alexander Postel ${ }^{4}(0)$, Anders Galatius ${ }^{5} \oplus$, \\ Line Anker Kyhn ${ }^{5}$, Charlotte Bie Thøstesen ${ }^{6}$, Sara Persson ${ }^{7}$, Ursula Siebert ${ }^{2} \mathbb{D}$, Morten Tange Olsen ${ }^{1} \mathbb{1}$ \\ and Paul Becher ${ }^{4, *}$ (D)
}

1 Evolutionary Genomics Section, GLOBE, University of Copenhagen, Øster Farimagsgade 5, 1353 Copenhagen, Denmark; iben.stokholm@sund.ku.dk (I.S.); morten.olsen@sund.ku.dk (M.T.O.)

2 Institute for Terrestrial and Aquatic Wildlife Research, University of Veterinary Medicine Hannover, Werftstr. 6, 25761 Büsum, Germany; ursula.siebert@tiho-hannover.de

3 Institute for Medical Microbiology, Virology and Hygiene, University Medical Center Hamburg-Eppendorf, 20251 Hamburg, Germany; nfischer@uke.de

4 Institute of Virology, University of Veterinary Medicine Hannover, Bünteweg 17, 30559 Hannover, Germany; christine.baechlein@tiho-hannover.de (C.B.); alexander.postel@tiho-hannover.de (A.P.)

5 Marine Mammal Research, Department of Ecoscience, Aarhus University, Frederiksborgvej 399, 4000 Roskilde, Denmark; agj@ecos.au.dk (A.G.); 1ky@ecos.au.dk (L.A.K.)

6 Fisheries and Maritime Museum, Tarphagevej 2, 6710 Esbjerg, Denmark; bt@fimus.dk

7 Swedish Museum of Natural History, Department of Environmental Research and Monitoring, 10405 Stockholm, Sweden; sara.persson@nrm.se

* Correspondence: paul.becher@tiho-hannover.de

\section{check for}

updates

Citation: Stokholm, I.; Fischer, N.; Baechlein, C.; Postel, A.; Galatius, A.; Kyhn, L.A.; Thøstesen, C.B.; Persson, S.; Siebert, U.; Olsen, M.T.; et al. In the Search of Marine Pestiviruses: First Case of Phocoena Pestivirus in a Belt Sea Harbour Porpoise. Viruses 2022, 14, 161. https://doi.org/ 10.3390/v14010161

Academic Editors: Chao-Nan Lin and Peck Toung Ooi

Received: 21 December 2021

Accepted: 13 January 2022

Published: 17 January 2022

Publisher's Note: MDPI stays neutral with regard to jurisdictional claims in published maps and institutional affiliations.

Copyright: (c) 2022 by the authors. Licensee MDPI, Basel, Switzerland. This article is an open access article distributed under the terms and conditions of the Creative Commons Attribution (CC BY) license (https:// creativecommons.org/licenses/by/ $4.0 /)$.

\begin{abstract}
Pestiviruses are widespread pathogens causing severe acute and chronic diseases among terrestrial mammals. Recently, Phocoena pestivirus (PhoPeV) was described in harbour porpoises (Phocoena phocoena) of the North Sea, expanding the host range to marine mammals. While the role of the virus is unknown, intrauterine infections with the most closely related pestivirusesBungowannah pestivirus (BuPV) and Linda virus (LindaV)—can cause increased rates of abortions and deaths in young piglets. Such diseases could severely impact already vulnerable harbour porpoise populations. Here, we investigated the presence of PhoPeV in 77 harbour porpoises, 277 harbour seals (Phoca vitulina), grey seals (Halichoerus grypus) and ringed seals (Pusa hispida) collected in the Baltic Sea region between 2002 and 2019. The full genome sequence of a pestivirus was obtained from a juvenile female porpoise collected along the coast of Zealand in Denmark in 2011. The comparative Bayesian phylogenetic analyses revealed a close relationship between the new PhoPeV sequence and previously published North Sea sequences with a recent divergence from genotype 1 sequences between 2005 and 2009. Our findings provide further insight into the circulation of PhoPeV and expand the distribution from the North Sea to the Baltic Sea region with possible implications for the vulnerable Belt Sea and endangered Baltic Proper harbour porpoise populations.
\end{abstract}

Keywords: Phocoena pestivirus; Pinnipeds; cetaceans; marine mammals; viral phylogeny; Bayesian phylogenetic analysis; virus evolution

\section{Introduction}

Pestiviruses comprise highly diverse and widespread RNA viruses infecting a broad range of mammalian hosts [1,2]. So far, 11 species have been approved by the International Committee on Taxonomy of Viruses (ICTV) including important pathogens such as bovine vial diarrhea viruses (BVDV-1 and BVDV-2), classical swine fever virus (CSFV) and border disease virus (BDV) [3]. These viruses can result in severe diseases in farm animals and cause significant economic losses [4,5]. During the past decade, the genus Pestivirus has experienced a rapid expansion of known species with the detection of novel viruses within the orders of Artiodactyla, Chiroptera, Rodentia and Pholidota [6]. A number of 
novel pestiviruses have been detected in pigs [7], sheep and goats [8-12], bats (Scotophilus kuhli and Rhinolophus affini) [13,14], rodents [14,15], pangolins (Manis javanica and Manis pentadactyla) [16] and harbour porpoises (Phocoena phocoena) [17], suggesting a total number of 19 pestivirus species termed Pestivirus $A$ through Pestivirus $S$ [6]. The recent discovery of porpoise pestivirus (Phocoena pestivirus, $\mathrm{PhoPeV}$ ) expands the host range of pestiviruses from terrestrial to marine mammals.

Pestiviruses are enveloped positive-sense single-stranded RNA viruses with a genome length of $12,000-16,500$ nucleotides encompassing a single open reading frame (ORF). The ORF encodes 12 mature proteins (Npro , C, E ${ }^{\text {rns }}$, E1, E2, p7, NS2, NS3, NS4A, NS4B, NS5A and NS5B) and is flanked by untranslated regions (UTRs) at each end $[2,6,18]$. The recently described porpoise pestivirus lacks the coding region of the N-terminal autoproteinase $\mathrm{N}^{\text {pro }}$ found in all other known pestiviruses [17]. The protein is implicated in the immunosuppression of pestivirus-infected cells by inhibiting the activation of the interferon-1 (IFN-1)-mediated cellular innate immune response [2]. It has been speculated that the loss of this specific genomic region might be due to an adaption to the cetacean immune system [17]. However, so far, there is no knowledge about the pathogenesis of PhoPeV and its potential impact on the health of porpoise populations. In terrestrial mammals, pestiviruses can be transmitted horizontally and vertically [2]. Horizontal transmission through direct and indirect contact with secretions and excretions can result in acute disease, immunosuppression or inapparent infections. In addition, vertical transmission to the fetus through intrauterine infection during gestation can result in abortions, fetal malformations and stillbirths as well as a specific acquired immunotolerance to the infecting virus and persistent infections of the offspring [2]. Persistently infected animals are unable to produce a humoral and cellular immune response against the virus, resulting in lifelong infection of seronegative animals with continuous shedding of large amounts of virus [2,18]. Moreover, different clinical conditions have been associated with infections of pregnant cattle, sheep and pigs by the widely distributed pestiviruses BVDV, BDV, CSFV and atypical porcine pestivirus (APPV), as well as by the unique Bungowannah pestivirus (BuPV) and Linda virus (LindaV) [2,7,19-21]. In pregnant sows infected with such pestiviruses, reproductive failure, as well as chronic wasting, myocarditis, congenital tremor and other nervous system dysfunctions of newborn piglets, have been described. Some of these traits could possibly occur in marine mammals infected by the novel porpoise pestivirus.

The porpoise populations inhabiting the greater Baltic Sea region comprise three populations; a North Sea-Skagerrak-Kattegat population, a Belt Sea population and a Baltic Proper population [22]. These are all impacted by bycatch, prey depletion and chemical and/or noise pollution, and the Belt Sea and Baltic Sea populations are listed as vulnerable and endangered, respectively [22-24]. As such, the introduction of a viral disease with a range of possible health implications could be devastating. However, to date, it is not known whether porpoise pestiviruses occur in the Baltic Sea region and if so, how they relate to porpoise pestivirus strains recently detected in the North Sea. Here, we conducted the first screening for Phocoena pestivirus in harbour porpoises collected in the Baltic Sea regions of Kattegat, the Danish Belt Sea, the Arkona Basin and the Bornholm Basin. In addition, to search for novel marine mammal hosts, we also screened harbour seals (Phoca vitulina), grey seals (Halichoerus grypus) and ringed seals (Pusa hispida) from the Baltic Sea and North Sea regions. We report the first isolation and full genome sequence of a pestivirus strain from a harbour porpoise collected along the coastline of Zealand in Denmark in 2011. Phylogenetic investigations were conducted on the divergence between the Baltic and recently published North Sea pestivirus sequences. Additional analyses of the divergence between porpoise pestivirus and related species (represented by Bungowannah virus and Linda virus) were made to further elucidate the origin of porpoise pestivirus and the transmission of pestiviruses between land and sea. 


\section{Materials and Methods}

\subsection{Sample Collection and Virus Detection}

Tissue samples (lung, spleen and reproduction organs) were collected from 77 porpoises stranded between 2007 and 2019 in the Baltic Sea and North Sea regions (Supplementary Table S1). In addition, to examining the potential occurrence of pestiviruses in sympatric marine mammal species, lung tissue from 85 harbour seals, 129 grey seals, and 63 ringed seals collected between 2002 and 2019 were included in the screenings (Supplementary Table S1). All samples were collected during necropsies and stored at $-20{ }^{\circ} \mathrm{C}$ (Danish and Swedish samples) or $-80^{\circ} \mathrm{C}$ (German samples).

\subsection{Virus Extraction and Screening}

Extractions of viral RNA from 77 harbour porpoises and 277 seals were made on individual lung $(n$ (porpoises) $=44, n$ (seals $)=173)$, pooled lung $(n$ (porpoises $)=13$, $n$ (seals $)=104)$ and mixed organ tissue $(n$ (porpoises $)=20)$ samples using the IndiMag Pathogen Kit w/o plastics (Cat.-No.: SP947257) with the homogenization of the tissue samples in the BeadMill 24 (Thermo Fisher) using the Lysing Matrix M (mpbio) and buffer RA1 + $\beta$-mercaptoethanol (Macherey/Nagel). Upon extraction, individual samples were combined in pools of $3-5$ individuals. To establish a broadly reactive PCR, three primer pairs were designed based on the available PhoPeV sequences, as well as sequences of the related BuPV and LindaV. As no PhoPeV genome-positive material was available, the primers were evaluated using BuPV genome-positive RNA preparations in a log10 dilution series. All three primer pairs resulted in specific amplicons. To be able to investigate the samples by real-time RT-PCR, analyses were performed with the QuantiTect SYBR Green RT-PCR Kit (Qiagen). The primers marinePV_204fw (5'-GTRCYACYGGWAAGGATCACCC-3') and marinePV_340rev (5'-CGCCGGCATCCTATCAGACTG-3') targeting a 137 base pairs (bp) fragment located within the $5^{\prime}$ UTR of the genome detected the BuPV genomic RNA with the highest sensitivity and were used for PhoPeV genome screening in the organ samples. Each reaction consisted of $12.5 \mu \mathrm{L}$ QuantiTect SYBR Green RT-PCR Mastermix, $1 \mu \mathrm{L}$ forward primer $(20 \mathrm{pmol})$ marinePV_204fw, $1 \mu \mathrm{L}$ reverse primer $(20 \mathrm{pmol})$ marinePV_340rev, $0.25 \mu \mathrm{L}$ reverse transcriptase, $5.25 \mu \mathrm{L} \mathrm{H}_{2} \mathrm{O}$ and $5 \mu \mathrm{L}$ RNA. Cycling conditions were set to $50{ }^{\circ} \mathrm{C}$ at $30 \mathrm{~min}, 95^{\circ} \mathrm{C}$ at $15 \mathrm{~min}, 40$ cycles of $15 \mathrm{~s}$ at $95^{\circ} \mathrm{C}, 30 \mathrm{~s}$ at $58^{\circ} \mathrm{C}$ and $30 \mathrm{~s}$ at $72{ }^{\circ} \mathrm{C}$.

\subsection{Full Genome Sequencing and Phylogenetic Analyses}

The genome sequence of the PhoPeV strain 43720 detected in lung, spleen and ovary tissue samples of a harbour porpoise collected in Denmark (Zealand) in 2011 (sample ID 43720; Supplementary Table S1; accession number: OK272505) was determined by next-generation sequencing on an Illumina HiSeq as previously described $[25,26]$. In order to investigate the origin and phylogenetic relationship of porpoise pestiviruses, different alignments were constructed based on the novel porpoise pestivirus sequence determined in this study and data available in public repositories. The first alignment was made to investigate the phylogenetic relationship between our novel porpoise pestivirus sequence, and the sequences detected in harbour porpoises from the North Sea. The alignment consisted of eight partial PhoPeV genomes covering the $5^{\prime}$ UTR and the genomic region coding for $\mathrm{C}, \mathrm{E}^{\mathrm{rns}}, \mathrm{E} 1$ and E2 proteins (2502 bp) (Supplementary Table S2). The second alignment was created to further elucidate the divergence between the related PhoPev, BuPV and LindaV sequences (Supplementary Table S3). This alignment covered the conserved regions 2 and 3, corresponding to the amino acid positions 1547-2321 and 2397-2688 numbered according to the reference sequence for BVDV-1 (strain SD-1, accession number: M96751) [3]. The alignments were selected based on initial substitution and recombination analyses of different alignments covering the partial and full-length genomes of available sequences (Supplementary Table S4).

The sequences for each alignment were imported and edited in Geneious version 9.1.8 [27], where the full and partial genomes were mapped to reference sequences of BVDV-1 (M96751) and BuPV (EF100713). The sequences were edited to the same reading 
frame and length corresponding to the region targeted. Alignments were generated using MUSCLE [28]. All nucleotide alignments were tested for substitution saturation and recombination prior to the Bayesian phylogenetic analyses. Recombination analyses were conducted in GARD [29] using the datamonkey.org server [30] with general discrete site-tosite variation and three rate classes using the HKY 85 likelihood ratio test to determine the significance of possible recombination sites (www.datamonkey.org). Substitution saturation tests were first performed using DAMBE and by evaluating plots of transitions and transversions prepared in R v.4 [31] using the packages "ape" [32] and "phylotools" [33] with the code adopted from Knudsen et al. (Supplementary Figures S1 and S2) [34]. As substitution saturation was detected on the 3rd codon position, for the second alignment (Supplementary Figure S2), all 3rd codon positions were removed using FASconCAT-G v1.02 [35] (2134 bp) to exclude potential bias.

The two nucleotide alignments were partitioned into 1st, 2nd and 3rd codon positions and 1st, 2nd codon positions, respectively and imported to BEAST v2.6.3 [36], where divergence time estimates and phylogenetic trees were generated using tip dates (sampling years) as time calibrations. Initial analyses with ModelTest-NG found TN93 (Tamura-Nei, 93) to be the best fit for the porpoise pestivirus alignment and TIM2 to be the best fit for the porpoise, BuPV and LindaV pestivirus alignments (Supplementary Table S5). Initial BEAST analyses were conducted on nucleotide and amino acid alignments with a strict clock with a chain length of 20,000,000 Markov chain Monte Carlo (MCMC) (Supplementary Table S6). Model selection was made based on marginal likelihood values generated through the path sampling analyses of different combinations of clock models (strict clock, lognormal relaxed clock and exponential clock) [37] and different tree models (coalescence constant, coalescence exponential growth, coalescence Bayesian skyline and birth death model) [38-40]. The analyses were made using a chain length of 1,000,000 generations, an alpha set to 0.3 , a burn-in percentage of $50 \%$, a pre-burn-in of 10,000 and a number of steps set to 120. In addition, the BEAST analyses of selected alignments were run with an MCMC chain length set to 200,000,000 generations, with sampling every 2000 iterations and a burn-in percentage of 10\%. All log files were evaluated in Tracer v1.6.0 [41] to ensure an effective sample size (ESS) of $>200$ for all parameters. Finally, test runs sampling from the prior setup and excluding the alignment data were evaluated to ensure that the results were not an artifact of disagreements among the priors comprising the model.

\section{Results and Discussion}

\subsection{PhoPeV Pestivirus Detected in the Baltic Sea Region}

In this study, we screened for porpoise pestivirus in 77 harbour porpoises and 277 seals collected along the Baltic and North Sea coastlines of Denmark, Germany and Sweden between 2002 and 2019 (Supplementary Table S1). One positive case was detected among the harbour porpoises; a juvenile female (ID 43720) found stranded on the coast of Zealand, Denmark, on the 4 February 2011 (Figure 1 and Supplementary Table S1). This finding represents the first detection of $\mathrm{PhoPeV}$ in the Baltic Sea region and expands the distribution range of porpoise pestiviruses from the North Sea harbour porpoise population to the Danish Belt Sea population. None of the seals tested positive for a PhoPeV-related pestivirus. However, the recent expansions of the pestivirus host range from Artiodactyla to Chiroptera, Rodentia and Pholidota suggest that it is broader than previously anticipated. As such, it remains to be tested if more distantly related pestiviruses circulate among other marine mammal species.

Based on previous findings of viral RNA among animals collected in 2001, 2003-2005, 2008 and 2012 [17], it seems reasonable to assume that PhoPeV was widespread in the North Sea between 2001 and 2012 or possibly circulated as an endemic virus among harbour porpoises of the area. In our study, 27 animals including one individual from the North Sea originate from this period (Figure 1 and Supplementary Table S1). No positive animals were found among the 49 harbour porpoises collected between 2017 and 2019, but it is uncertain whether this owe to the small sample size or if the virus was not circulating in the 
population during this time period. All tissues analysed in this study were collected from stranded animals, and as such, this survey cannot contribute to prevalence estimations in the healthy population.

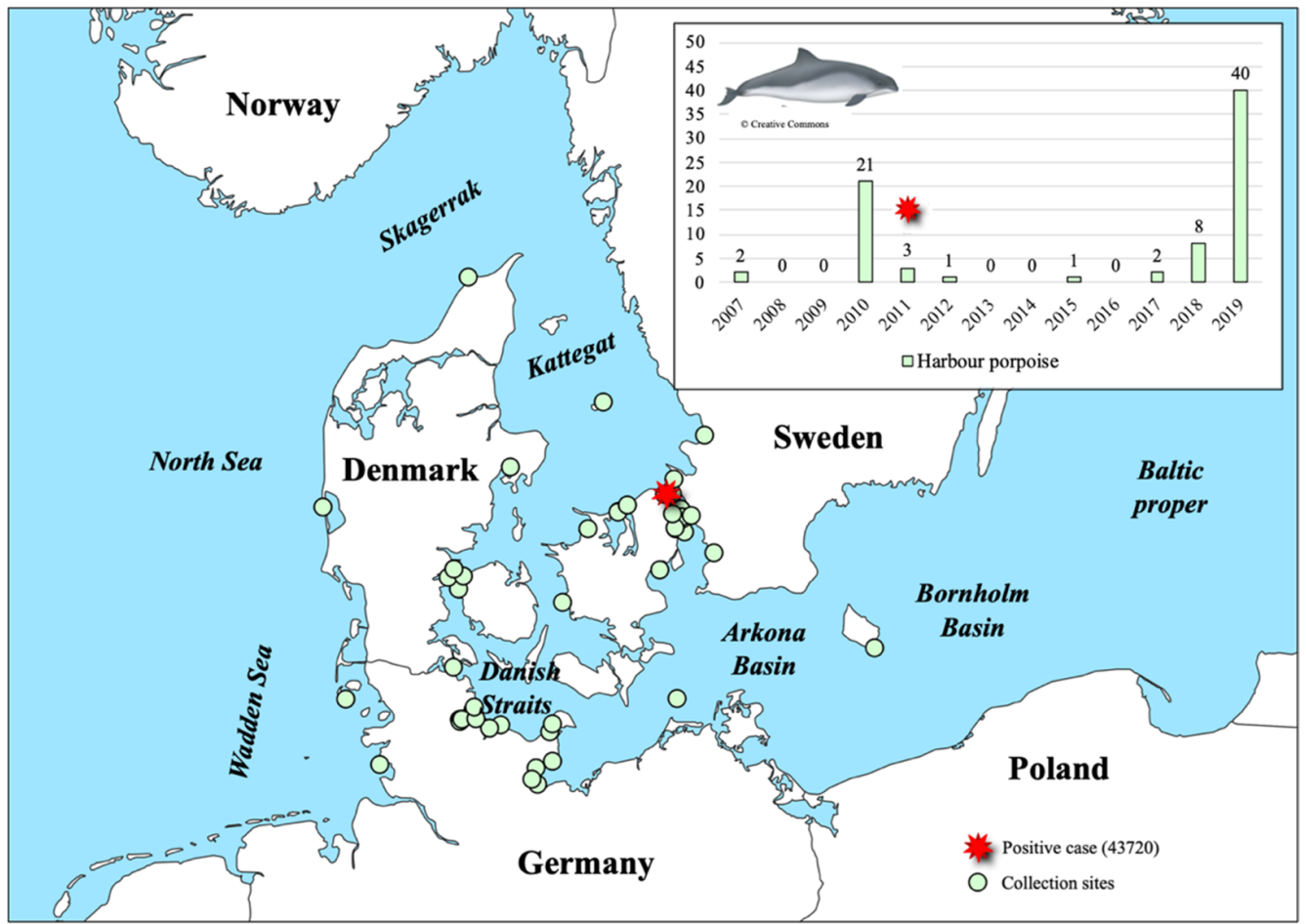

Figure 1. Map and histogram illustrating collection sites (green dots) and distribution of 77 harbour porpoises screened per year between 2007 and 2019. The red star indicates the collection site of the Phocoena pestivirus (PhoPeV)-positive harbour porpoise (ID: 43720 and collection date: 4 February 2011).

The PhoPeV-positive individual reported in this study was well nourished with a blubber thickness of 27-36 mm and a length and a weight of $117 \mathrm{~cm}$ and $31.2 \mathrm{~kg}$, respectively. No signs of inflammation were noted during necropsy. Based on the findings of foam in the bronchi and trachea and fresh wounds on its fins and body, it was concluded that the most likely cause of death was drowning by entanglement. The detection of pestivirus RNA in lung (Ct: 20.9), spleen (Ct: 20.8) and reproductive organs (Ct: 22.1) can be the result of acute or persistent pestivirus infection. Taking into account the lack of necropsy findings and a considerably higher probability of encountering lifelong persistent pestivirus infections, a persistent infection appears more likely. As persistently infected animals stay seronegative, future surveys based on antibody prevalence should be supported by screenings for viral RNA or antigens.

\subsection{Determination of the Genome Sequence of PhoPeV Strain 43720 and the Phylogenetic Relationship among Porpoise Pestiviruses}

The genome sequence of the PhoPeV strain 43720 was determined by high-throughput sequencing using pooled RNA extracted from lung, spleen and ovary tissue samples of a harbour porpoise collected in Denmark (Zealand) in 2011 (accession number: OK272505). The obtained sequence (mean coverage: 2800) of PhoPeV strain 43720 comprises 11,872 nucleotides (nt). It contains one large ORF and 5' and $3^{\prime}$ UTRs of $374 \mathrm{nt}$ and $212 \mathrm{nt}$, respectively. The ORF is 11,286 nt long and encodes a polyprotein encompassing 3761 amino acids. The comparison with available complete pestivirus sequences including previously reported sequences of PhoPeV strains from the North Sea revealed that the ORF and 3' UTR sequences of PhoPeV strain 43720 are complete, while eight highly conserved nucleotides 
escaped the sequencing at the $5^{\prime}$ terminus of the viral genome. The comparison of genome sequences revealed that PhoPeV strain 43720 is most closely related to other PhoPeV genomic sequences ( $>97 \%$ identity), whereas the identities with all other pestivirus sequences were below 71\%. As previously described for the PhoPeV strains obtained from animals stranded at the North Sea, the newly generated sequence of PhoPeV strain 43720 lacks the pestivirus-specific $\mathrm{N}^{\mathrm{pr}}$-encoding sequence [17].

Bayesian phylogenetic analyses were conducted on all currently available PhoPeV sequences to elucidate the origin of the porpoise pestivirus we detected in the Baltic Sea region. The phylogenetic analysis placed the new sequence together with genotype 1 sequences of harbour porpoises collected in the Dutch part of the Wadden Sea in 2001, 2005, 2008, 2012 and 2014. The estimated time to the most recent common ancestor (tMRCA) varied, depending on the model setup with the mean estimates ranging from 1880 to 1996 (Supplementary Table S7). However, the likelihood values provided the highest support for model 8 with an estimated tMRCA of all known PhoPeV sequences between 1986 and 2001 and a recent divergence ( 2009 (2006-2011)) between our Belt Sea and previously published North Sea PhoPeV sequences obtained from animals collected between 2005 and 2009 (Figure 2 and Supplementary Table S7). Thus, our results support a rather recent exchange of PhoPeV between North Sea harbour porpoises and the harbour porpoise collected in the Baltic Sea region. The finding is significant, as the introductions or continuous circulation of PhoPeV could be yet another factor causing declines in the vulnerable Belt Sea and endangered Baltic Proper harbour porpoise populations.

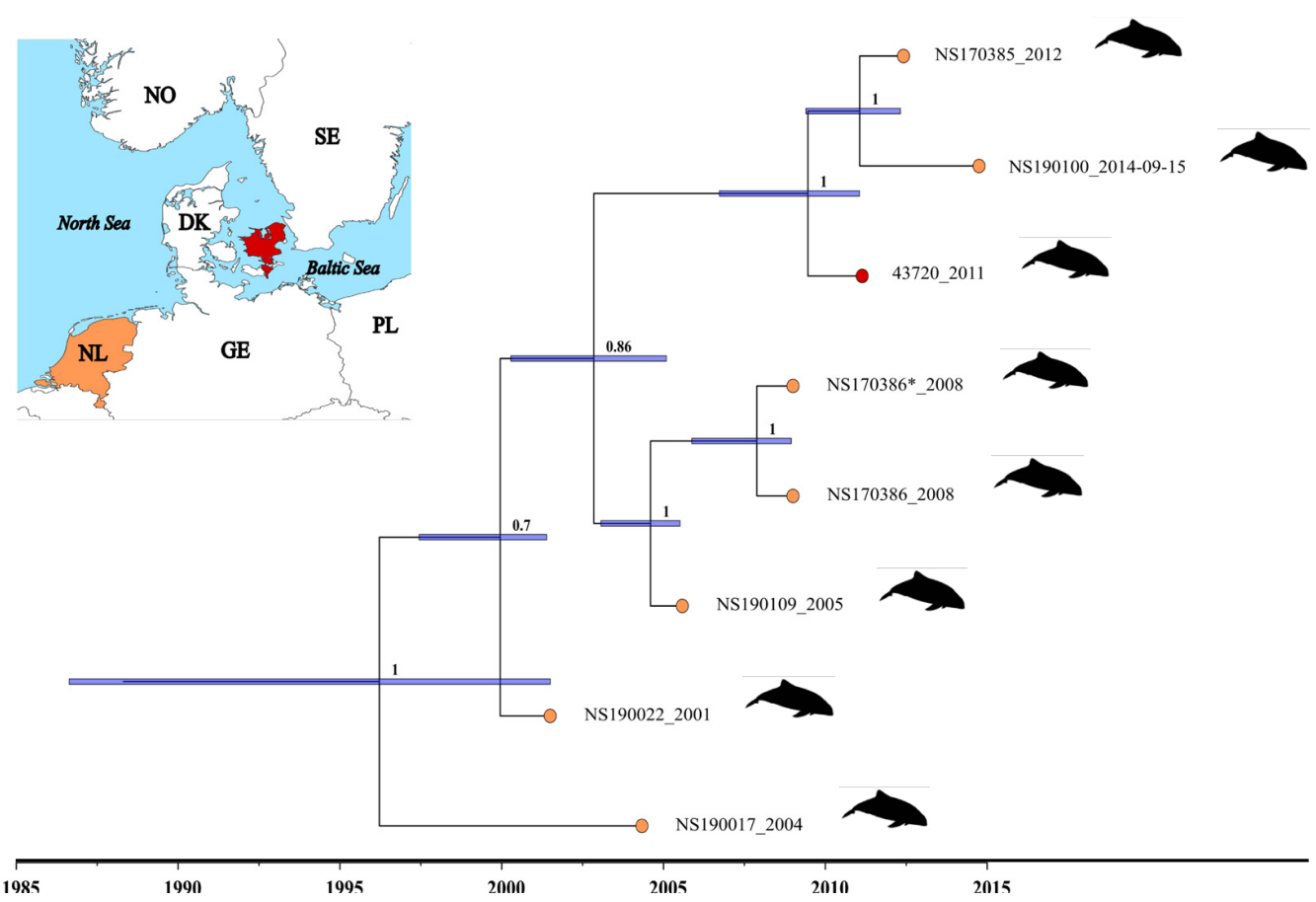

Figure 2. Time-calibrated Bayesian phylogenetic tree of the Baltic PhoPeV sequence $(43,720)$ described here and seven recently published PhoPeV sequences from the Netherlands collected between 2001 and 2014 (GenBank accession number: MK910230-37). The tree was generated in BEAST 2.5.2 based on the concatenated nucleotide alignment comprising the $5^{\prime}$ untranslated regions (UTR) and the regions coding for $\mathrm{C}, \mathrm{E}^{\mathrm{rns}}, \mathrm{E} 1$ and $\mathrm{E} 2$ proteins. The tree was generated using a relaxed exponential clock and a coalescence constant tree prior (Supplementary Table S1). The colour-coded circles at the tips indicate the countries (the red colour represents Denmark, and the orange colour represents the Netherlands) where the animals were collected. The node bars represent the highest posterior density (HPD) interval of the time estimates, while the tree support is shown as posterior values at each node. 


\subsection{Phylogenetic Relationship of Terrestrial and Marine Pestiviruses}

Investigations of the phylogenetic relationship and divergence between porpoise pestivirus and the most closely related terrestrial BuPV and LindaV sequences were conducted to shed light on the origin of porpoise pestivirus and the dispersal of pestiviruses from terrestrial to marine hosts. Unfortunately, the detection of recombination and substitution saturation in several parts of the genome limited our final alignment to cover the 1st and 2nd codon positions of the conserved regions 2 and 3 . The phylogenetic analyses using different models and clocks yielded inconsistent results with regard to the tree topology and divergence times (Supplementary Table S8; Figure 3A,B). The path sampling analyses found the highest likelihood values for the relaxed lognormal clock in combination with a coalescence exponential tree model (Supplementary Table S8). However, the topology and divergence estimates from this model suggested a close relationship between PhoPeV and LindaV and a divergence time in the late 20th century (Figure 3A; Supplementary Table S8), which is inconsistent with previous analyses of pestivirus evolution [16,17]. To check for effects of data reductions associated with the removal of the 3rd codon positions, we compared analyses including and excluding the substitution-saturated 3rd codon positions separately but found no significant difference in the divergence estimates and topology. Moreover, test runs of the relaxed clock analyses only sampling from the prior provided similar time estimates as runs with data, with no apparent discrepancies in the model setup that could artificially drive these early estimates. However, the data set is limited to just four PhoPeV, three BuPV and two LindaV sequences which taken together with the alignments' limited coverage of variable regions may contain insufficient information for the relaxed clock models to be informative. In contrast to the results of the relaxed clock model, the strict clock model supported a close relationship between BuPV and LindaV with porpoise pestivirus as an outgroup to these (Figure 3B), corresponding to previously reported phylogenetic relationships between the viruses [16,17]. The divergence time estimates provided by the strict clock model varied, depending on the input data and the tree model, but most pointed to a divergence of $\mathrm{PhoPeV}$ from other pestiviruses between the 17th and the 20th centuries (Figure 3; Supplementary Tables S6 and S8). However, based on the path sampling analysis providing the highest support for a relaxed clock and the discussed implications of the limited data set, we regarded these results as inconclusive.

$\mathrm{PhoPeV}, \mathrm{BuPV}$ and LindaV are each other's closest relatives among known pestiviruses but only share $60 \%$ homology at the amino acid level [17]. Thus, future studies on the origin of marine pestiviruses would benefit from the detection and analysis of more closely related strains, i.e., "missing links", which would allow for the creation of data sets holding less recombination and substitution saturation and aid the generation of more accurate time estimates. Moreover, recent analyses of the divergence between related morbilliviruses, Measles virus and Rinderpest virus, utilized codon-based models and ancient virus strains to obtain more stable and reliable results [42,43]. While the detection of ancient wildlife pestiviruses currently seems unlikely, future investigations into the evolutionary history of these viruses would benefit from an extended data set including additional genome sequences representing a larger number of diverse porpoise, Bungowannah and Linda pestiviruses. Such additional analyses could be carried out on full-genome codon-based models (excluding recombinant sites) [43]. As seen with other studies on virus evolution, it is likely that the addition of additional, novel and historical strains will similarly push the estimated divergence between terrestrial and marine pestiviruses back in time $[43,44]$. 


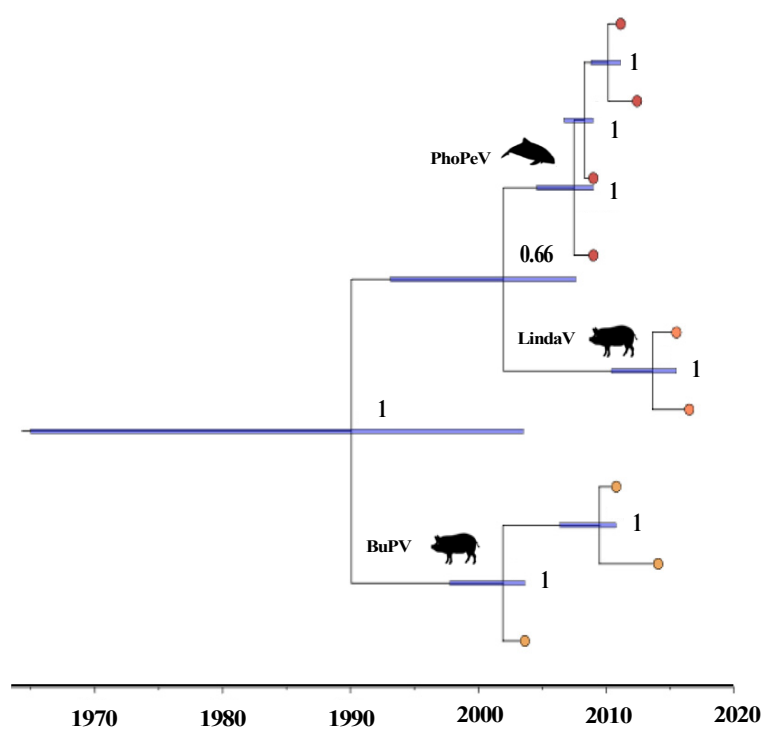

(A)

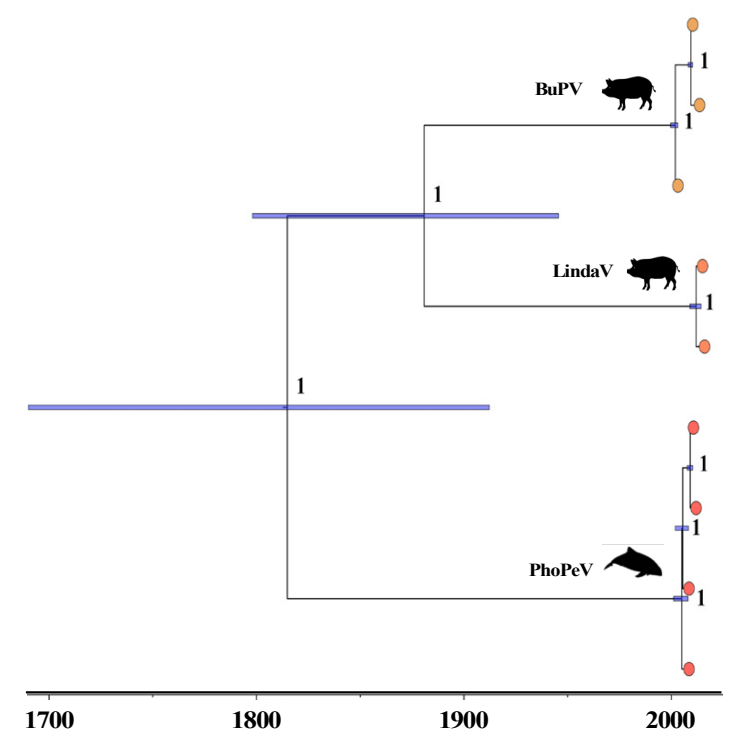

(B)

Figure 3. Time-calibrated Bayesian phylogenetic trees of available PhoPeV, Bungowannah pestivirus (BuPV) and Linda virus (LindaV) sequences (Supplementary Table S3) covering conserved regions 2 and 3 corresponding to the amino acid positions 1547-2321 and 2397-2688 numbered according to the reference sequence for BVDV-1 (strain SD-1) (accession number: M96751) [3]. The trees were generated in BEAST 2.5.2 under two different model setups. (A) Tree generated using a relaxed clock lognormal and an exponential coalescence tree prior. This tree was the most strongly supported tree based on the path sampling analysis (Supplementary Table S8). (B) Tree generated using a strict clock and a Bayesian skyline tree prior. This model set up showed the strongest support among models using a strict clock (Supplementary Table S8). The node bars represent the highest posterior density (HPD) interval of the time estimates, while the tree support is shown as posterior values at each node.

\subsection{Pestiviruses in the Marine Ecosystem}

The detection of PhoPeV in a harbour porpoise from Zealand, Denmark, expands the distribution range from the North Sea to the Belt Sea populations. Its presence gives cause for concern, as infections might negatively impact the reproductivity of the vulnerable Belt Sea and endangered Baltic Proper harbour porpoises. The Belt Sea case could be a result of a single introduction of a widespread virus or the observation of an endemically circulating virus detected in a single individual. Additional findings of virus, viral genomes, antigens or antibodies are needed to further evaluate the circulation and presence of $\mathrm{PhoPeV}$ among harbour porpoise populations in this region and elsewhere to determine the impact of the virus. Therefore, we suggest that harbour porpoise health-monitoring programs should include this putative pathogen.

Too little is currently known about $\mathrm{PhoPeV}$ to establish the origin and dispersal of the virus. While only recently detected in porpoises, pestiviruses might have been circulating in the marine ecosystem for centuries. The absence of the $\mathrm{N}^{\text {pro }}$ protein-coding region in the $\mathrm{PhoPeV}$ genome clearly distinguishes it from other pestiviruses and suggests that there might be yet unidentified "missing links" among PhoPeV and related pestiviruses. This assumption is further supported by the disparate distribution of BuPV and LindaV, which represent unique viruses and have only been found in pig herds on geographically distant farms in Australia and Austria, respectively [7,45], as well as by the recent discovery of the related Dongyang pangolin virus (DYPV) in China [16]. It has been speculated that human-mediated dispersal by livestock transport or a contaminated vaccine could have promoted virus spread across large distances [46]. However, as BuPV and LindaV have not been detected in other domestic pig herds or in wild boar populations, it seems more likely 
that the emergence of these two porcine viruses were caused by spillover from unknown (wild) reservoirs [47-49]. A more complete understanding of the origin, evolution and spread of PhoPeV, BuPV and LindaV awaits the discovery of related pestiviruses in marine and terrestrial mammals.

\subsection{Isolation of PhoPeV 43720 and Future Studies}

It has been reported that PhoPeV from the North Sea could be isolated on porcine and bovine cell cultures [17]. While initial attempts to isolate the Baltic PhoPeV strain 43720 failed, virus isolation was finally successful by the inoculation of porcine SPEV kidney cells with dilutions of organ suspensions. The availability of PhoPeV isolates will facilitate the establishment of assays for the detection of PhoPeV-specific antibodies and allow the determination of prevalence and cross-reactivity with related pestiviruses. Future studies will focus on the characterization of biological properties of PhoPeV and include investigations on the host range, viral entry and replication in tissue culture cells to enhance our knowledge about the biology of this first marine pestivirus.

Supplementary Materials: The following supporting information can be downloaded at: https: / / www.mdpi.com/article/10.3390/v14010161/s1, Figure S1: Two different substitution saturation plots for the PhoPeV alignment and Figure S2: Two different substitution saturation plots for the PhoPeV, BuPV, LindaV alignment. Table S1: A complete list of the harbour porpoises, harbour seals, grey seals and ringed seals screened throughout this study, Table S2: Information on sequences included in the PhoPeV phylogeny, Table S3: Information on sequences used in the PhoPeV, BuPV and LindaV analyses, Table S4: Overview of initial alignments tested for recombination and substitution saturation including coverage, number of sequences and sequence length, Table S5: Results from the Modeltest-NG indicating the best fit substitution model for the data sets based on the AIC and BIC, Table S6: Results of initial BEAST tests of nucleotide and amino acid alignments of PhoPeV, BuPeV and LindaV sequences run with a strict clock and 20,000,000 MCMC, Table S7: Results of path sampling analyses of the PhoPeV alignment evaluating the best fit model for the data by the calculation of likelihood scores, Table S8: Results of path sampling analyses of the PhoPeV, BuPV and Linda $\mathrm{V}$ alignment evaluating the best fit model for the data by the calculation of likelihood scores.

Author Contributions: I.S., U.S., M.T.O. and P.B. conceived and designed the study; U.S., M.T.O. and P.B. provided funding; A.G., L.A.K., C.B.T., S.P. and U.S. provided samples; I.S., N.F., C.B., A.P., P.B. and M.T.O. performed the analyses; I.S., U.S., M.T.O. and P.B. drafted the manuscript. All authors have read and agreed to the published version of the manuscript.

Funding: This work was supported by matching funding from the University of Copenhagen, Denmark to I.S. and M.T.O., the University of Veterinary Medicine Hannover to I.S. and the "BONUS BALTHEALTH" project awarded to U.S. and M.T.O. BONUS BALTHEALTH received funding from BONUS (Art. 185). BONUS BALTHEALTH was funded jointly by the E.U., Innovation Fund Denmark (grants 6180-00001B and 6180-00002B), Forschungszentrum Jülich GmbH, German Federal Ministry of Education and Research (grant FKZ 03F0767A), Academy of Finland (grant 311966) and Swedish Foundation for Strategic Environmental Research (MISTRA).

Institutional Review Board Statement: Ethical review and approval were waived for this study, due to the exclusive use of stored samples collected during the necropsies of stranded animals.

Informed Consent Statement: Not applicable.

Data Availability Statement: The data sets are submitted together with the study. The novel PhoPeV sequence has been submitted to GenBank (accession number: OK272505).

Acknowledgments: We thank Steen Wilhelm Knudsen for advice on the phylogenetic analyses, Inga Grotha and Ester Barthel for advice on RT-qPCR amplifications, as well as the field personal involved in necropsies and sample collections at museums, NGOs, nature agencies and research institutes in Denmark, Germany and Sweden.

Conflicts of Interest: The authors declare no conflict of interest. 


\section{References}

1. Simmonds, P.; Becher, P.; Bukh, J.; Gould, E.A.; Meyers, G.; Monath, T.; Muerhoff, S.; Pletnev, A.; Rico-Hesse, R.; Smith, D.B.; et al. ICTV Virus Taxonomy Profile: Flaviviridae. J. Gen. Virol. 2017, 98, 2-3. [CrossRef]

2. Becher, P.; Moennig, V.; Tautz, N. Bovine Viral Diarrhea, Border Disease, and Classical Swine Fever Viruses; Elsevier Ltd.: Amsterdam, The Netherlands, 2020. [CrossRef]

3. Smith, D.B.; Meyers, G.; Bukh, J.; Gould, E.A.; Monath, T.; Muerhoff, A.S.; Pletnev, A.; Rico-Hesse, R.; Stapleton, J.T.; Simmonds, P.; et al. Proposed Revision to the Taxonomy of the Genus Pestivirus, Family Flaviviridae. J. Gen. Virol. 2017, 98, $2106-2112$. [CrossRef]

4. Moennig, V.; Becher, P. Pestivirus Control Programs: How Far Have We Come and Where Are We Going? Anim. Heal. Res. Rev. 2015, 16, 83-87. [CrossRef]

5. Moennig, V.; Becher, P. Control of Bovine Viral Diarrhea. Pathogens 2018, 7, 29. [CrossRef]

6. Postel, A.; Smith, D.B.; Becher, P. Proposed Update to the Taxonomy of Pestiviruses: Eight Additional Species within the Genus Pestivirus, Family Flaviviridae. Viruses 2021, 13, 1542. [CrossRef]

7. Lamp, B.; Schwarz, L.; Högler, S.; Riedel, C.; Sinn, L.; Rebel-Bauder, B.; Weissenböck, H.; Ladinig, A.; Rümenapf, T. Novel Pestivirus Species in Pigs, Austria, 2015. Emerg. Infect. Dis. 2017, 23, 1176-1179. [CrossRef]

8. Thabti, F.; Letellier, C.; Hammami, S.; Pépin, M.; Ribière, M.; Mesplède, A.; Kerkhofs, P.; Russo, P. Detection of a Novel Border Disease Virus Subgroup in Tunisian Sheep. Arch. Virol. 2005, 150, 215-229. [CrossRef]

9. Postel, A.; Schmeiser, S.; Oguzoglu, T.C.; Indenbirken, D.; Alawi, M.; Fischer, N.; Grundhoff, A.; Becher, P. Close Relationship of Ruminant Pestiviruses and Classical Swine Fever Virus. Emerg. Infect. Dis. 2015, 21, 668-672. [CrossRef]

10. Ciulli, S.; Purpari, G.; Agnello, S.; Di Marco, P.; Di Bella, S.; Volpe, E.; Mira, F.; de Aguiar Saldanha Pinheiro, A.C.; Vullo, S.; Guercio, A. Evidence for Tunisian-Like Pestiviruses Presence in Small Ruminants in Italy Since 2007. Transbound. Emerg. Dis. 2017, 64, 1243-1253. [CrossRef]

11. Sozzi, E.; Lavazza, A.; Gaffuri, A.; Bencetti, F.C.; Prosperi, A.; Lelli, D.; Chiapponi, C.; Moreno, A. Isolation and Full-Length Sequence Analysis of a Pestivirus from Aborted Lamb Fetuses in Italy. Viruses 2019, 11, 744. [CrossRef]

12. Meyer, D.; Postel, A.; Wiedemann, A.; Cagatay, G.N.; Ciulli, S.; Guercio, A.; Becher, P. Comparative Analysis of Tunisian Sheep-like Virus, Bungowannah Virus and Border Disease Virus Infection in the Porcine Host. Viruses 2021, 13, 1539. [CrossRef]

13. Wu, Z.; Ren, X.; Yang, L.; Hu, Y.; Yang, J.; He, G.; Zhang, J.; Dong, J.; Sun, L.; Du, J.; et al. Virome Analysis for Identification of Novel Mammalian Viruses in Bat Species from Chinese Provinces. J. Virol. 2012, 86, 10999-11012. [CrossRef]

14. Wu, Z.; Liu, B.; Du, J.; Zhang, J.; Lu, L.; Zhu, G.; Han, Y.; Su, H.; Yang, L.; Zhang, S.; et al. Discovery of Diverse Rodent and Bat Pestiviruses with Distinct Genomic and Phylogenetic Characteristics in Several Chinese Provinces. Front. Microbiol. 2018, 9, 1-8. [CrossRef]

15. Firth, C.; Bhat, M.; Firth, M.A.; Williams, S.H.; Frye, M.J.; Simmonds, P.; Conte, J.M.; Ng, J.; Garcia, J.; Bhuva, N.P.; et al. Detection of Zoonotic Pathogens and Characterization of Novel Viruses Carried by Commensal Rattus Norvegicus in New York City. $m$ Bio 2014, 5, 1-16. [CrossRef]

16. Gao, W.H.; Lin, X.D.; Chen, Y.M.; Xie, C.G.; Tan, Z.Z.; Zhou, J.J.; Chen, S.; Holmes, E.C.; Zhang, Y.Z. Newly Identified Viral Genomes in Pangolins with Fatal Disease. Virus Evol. 2020, 6, 1-10. [CrossRef]

17. Jo, W.K.; van Elk, C.; van de Bildt, M.; van Run, P.; Petry, M.; Jesse, S.T.; Jung, K.; Ludlow, M.; Kuiken, T.; Osterhaus, A. An Evolutionary Divergent Pestivirus Lacking the Npro Gene Systemically Infects a Whale Species. Emerg. Microbes Infect. 2019, 8, 1383-1392. [CrossRef]

18. Tautz, N.; Tews, B.A.; Meyers, G. The Molecular Biology of Pestiviruses, 1st ed.; Elsevier Inc.: Amsterdam, The Netherlands, 2015; Volume 93. [CrossRef]

19. De Groof, A.; Deijs, M.; Guelen, L.; Van Grinsven, L.; Van Os-Galdos, L.; Vogels, W.; Derks, C.; Cruijsen, T.; Geurts, V.; Vrijenhoek, M.; et al. Atypical Porcine Pestivirus: A Possible Cause of Congenital Tremor Type A-II in Newborn Piglets. Viruses 2016, 8, 271. [CrossRef]

20. Postel, A.; Hansmann, F.; Baechlein, C.; Fischer, N.; Alawi, M.; Grundhoff, A.; Derking, S.; Tenhündfeld, J.; Pfankuche, V.M.; Herder, V.; et al. Presence of Atypical Porcine Pestivirus (APPV) Genomes in Newborn Piglets Correlates with Congenital Tremor. Sci. Rep. 2016, 6, 1-9. [CrossRef]

21. Finlaison, D.S.; Kirkland, P.D. The Outcome of Porcine Foetal Infection with Bungowannah Virus Is Dependent on the Stage of Gestation at Which Infection Occurs. Part 2: Clinical Signs and Gross Pathology. Viruses 2020, 12, 873. [CrossRef]

22. Sveegaard, S.; Galatius, A.; Dietz, R.; Kyhn, L.; Koblitz, J.C.; Amundin, M.; Nabe-Nielsen, J.; Sinding, M.H.S.; Andersen, L.W.; Teilmann, J. Defining Management Units for Cetaceans by Combining Genetics, Morphology, Acoustics and Satellite Tracking. Glob. Ecol. Conserv. 2015, 3, 839-850. [CrossRef]

23. ASCOBANS. Draft Recovery Plan for Baltic Harbour Porpoises (Jastarnia Plan); ASCOBANS: Bonn, Germany, 2002.

24. Benke, H.; Bräger, S.; Dähne, M.; Gallus, A.; Hansen, S.; Honnef, C.G.; Jabbusch, M.; Koblitz, J.C.; Krügel, K.; Liebschner, A.; et al. Baltic Sea Harbour Porpoise Populations: Status and Conservation Needs Derived from Recent Survey Results. Mar. Ecol. Prog. Ser. 2014, 495, 275-290. [CrossRef]

25. Fischer, N.; Indenbirken, D.; Meyer, T.; Lütgehetmann, M.; Lellek, H.; Spohn, M.; Aepfelbacher, M.; Alawi, M.; Grundhoff, A. Evaluation of Unbiased Next-Generation Sequencing of RNA (RNA-Seq) as a Diagnostic Method in Influenza Virus-Positive Respiratory Samples. J. Clin. Microbiol. 2015, 53, 2238-2250. [CrossRef] 
26. Alawi, M.; Burkhardt, L.; Indenbirken, D.; Reumann, K.; Christopeit, M.; Kröger, N.; Lütgehetmann, M.; Aepfelbacher, M.; Fischer, N.; Grundhoff, A. DAMIAN: An Open Source Bioinformatics Tool for Fast, Systematic and Cohort Based Analysis of Microorganisms in Diagnostic Samples. Sci. Rep. 2019, 9, 1-17. [CrossRef]

27. Kearse, M.; Moir, R.; Wilson, A.; Stones-Havas, S.; Cheung, M.; Sturrock, S.; Buxton, S.; Cooper, A.; Markowitz, S.; Duran, C.; et al. Geneious Basic: An Integrated and Extendable Desktop Software Platform for the Organization and Analysis of Sequence Data. Bioinformatics 2012, 28, 1647-1649. [CrossRef]

28. Edgar, R.C. MUSCLE: Multiple Sequence Alignment with High Accuracy and High Throughput. Nucleic Acids Res. 2004, 32, 1792-1797. [CrossRef]

29. Pond, S.L.K.; Posada, D.; Gravenor, M.B.; Woelk, C.H.; Frost, S.D.W. Automated Phylogenetic Detection of Recombination Using a Genetic Algorithm. Mol. Biol. Evol. 2006, 23, 1891-1901. [CrossRef]

30. Delport, W.; Poon, A.F.Y.; Frost, S.D.W.; Kosakovsky Pond, S.L. Datamonkey 2010: A Suite of Phylogenetic Analysis Tools for Evolutionary Biology. Bioinformatics 2010, 26, 2455-2457. [CrossRef]

31. R Core Team. R: A Language and Environment for Statistical Computing; R Foundation for Statistical Computing: Vienna, Austria, 2020.

32. Paradis, E.; Schliep, K. Ape 5.0: An Environment for Modern Phylogenetics and Evolutionary Analyses in R. Bioinformatics 2019, 35, 526-528. [CrossRef]

33. Zhang, J.; Pei, N.; Mi, X. Phylotools: Phylogenetic Tools for Eco-Phylogenetics. v. 0.2.2. 2017. Available online: https: / / cran.r-project.org / package=phylotools.

34. Knudsen, S.W.; Choat, J.H.; Clements, K.D. The Herbivorous Fish Family Kyphosidae (Teleostei: Perciformes) Represents a Recent Radiation from Higher Latitudes. J. Biogeogr. 2019, 46, 2067-2080. [CrossRef]

35. Kück, P.; Meusemann, K. FASconCAT; Zoologisches Forschungsmuseum: Bonn, Germany, 2010.

36. Bouckaert, R.; Heled, J.; Kühnert, D.; Vaughan, T.; Wu, C.H.; Xie, D.; Suchard, M.A.; Rambaut, A.; Drummond, A.J. BEAST 2: A Software Platform for Bayesian Evolutionary Analysis. PLoS Comput. Biol. 2014, 10, 1-6. [CrossRef]

37. Drummond, A.J.; Ho, S.Y.W.; Phillips, M.J.; Rambaut, A. Relaxed Phylogenetics and Dating with Confidence. PLoS Biol. 2006, 4, 699-710. [CrossRef]

38. Drummond, A.J.; Bouckaert, R.R. Bayesian Evolutionary Analysis with BEAST; Cambridge University Press: Cambridge, UK, 2015. [CrossRef]

39. Drummond, A.J.; Rambaut, A. BEAST: Bayesian Evolutionary Analysis by Sampling Trees. BMC Evol. Biol. 2007, 7, 214. [CrossRef]

40. Drummond, A.J.; Rambaut, A.; Shapiro, B.; Pybus, O.G. Bayesian Coalescent Inference of Past Population Dynamics from Molecular Sequences. Mol. Biol. Evol. 2005, 22, 1185-1192. [CrossRef]

41. Rambaut, A.; Surchard, M.A.; Xie, D.; Drummond, A.J. Tracer v1.6. 2014. Available online: http://beast.bio.ed.ac.uk/Tracer.

42. Goldman, N.; Yang, Z. A Codon-Based Model of Nucleotide Substitution for Protein-Coding DNA Sequences. Mol. Biol. Evol. 1994, 11, 725-736. [CrossRef]

43. Düx, A.; Lequime, S.; Patrono, L.V.; Vrancken, B.; Boral, S.; Gogarten, J.F.; Hilbig, A.; Horst, D.; Merkel, K.; Prepoint, B.; et al. Measles Virus and Rinderpest Virus Divergence Dated to the Sixth Century BCE. Science 2020, 368, 1367-1370. [CrossRef]

44. Mühlemann, B.; Vinner, L.; Margaryan, A.; Wilhelmson, H.; de la Fuente Castro, C.; Allentoft, M.E.; de Barros Damgaard, P.; Hansen, A.J.; Holtsmark Nielsen, S.; Strand, L.M.; et al. Diverse Variola Virus (Smallpox) Strains Were Widespread in Northern Europe in the Viking Age. Science 2020, 369, 6502. [CrossRef]

45. McOrist, S.; Thornton, E.; Peake, A.; Walker, R.; Robson, S.; Finlaison, D.; Kirkland, P.; Reece, R.; Ross, A.; Walker, K.; et al. An Infectious Myocarditis Syndrome Affecting Late-Term and Neonatal Piglets. Aust. Vet. J. 2004, 82, 509-511.

46. Kirkland, P.D.; Read, A.J.; Frost, M.J.; Finlaison, D.S. Bungowannah Virus-A Probable New Species of Pestivirus-What Have We Found in the Last 10 Years? Anim. Heal. Res. Rev. 2015, 16, 60-63. [CrossRef]

47. Cagatay, G.N.; Antos, A.; Meyer, D.; Maistrelli, C.; Keuling, O.; Becher, P.; Postel, A. Frequent Infection of Wild Boar with Atypical Porcine Pestivirus (APPV). Transbound. Emerg. Dis. 2018, 65, 1087-1093. [CrossRef]

48. Michelitsch, A.; Dalmann, A.; Wernike, K.; Reimann, I.; Beer, M. Seroprevalences of Newly Discovered Porcine Pestiviruses in German Pig Farms. Vet. Sci. 2019, 6, 86. [CrossRef]

49. Kiesler, A.; Plankensteiner, J.; Schwarz, L.; Riedel, C.; Seitz, K.; Mötz, M.; Ladinig, A.; Lamp, B.; Rümenapf, T. Prevalence of Linda Virus Neutralizing Antibodies in the Austrian Pig Population. Viruses 2021, 13, 1001. [CrossRef] 